\title{
Características de la dieta en niños de 2 a 5 años según la recepción de programas alimentarios en el hogar en Argentina*
}

\section{Characteristics of the Diet for Children Aged 2 to 5 According to the Reception of Alimentary Programs at Home in Argentina}

\section{Características da dieta em crianças de 2 até 5 anos de acordo com a recepção de programas alimentares em casa na Argentina}

Fecha de recepción: 28-04-14 Fecha de aceptación: 09-05-14 Disponible en línea: 01-07-14 doi: 10.11144/Javeriana.rgyps13-27.cdna

Cómo citar este artículo:

Acosta L. Características de la dieta en niños de 2 a 5 años según la recepción de programas alimentarios en el hogar en Argentina. Rev. Gerenc. Polít. Salud. 2014; 13(27): 319-333. http://dx.doi.org/10.11144/Javeriana.rgyps13-27.cdna

Artículo de investigación basado en el trabajo de investigación para la obtención del grado de magíster en Salud Pública (Universidad Nacional de Córdoba, Argentina): Análisis de la Dieta en destinatarios de programas alimentarios en Argentina, en base a la Encuesta Nacional de Nutrición y Salud, 2005. Fecha de inicio: mayo de 2010; fecha de finalización: septiembre de 2013.

* Centro de Investigaciones y Estudios sobre Cultura y Sociedad (CIECS), Unidad Ejecutora del Consejo Nacional de Investigaciones Científicas y Técnicas (Conicet) - Universidad Nacional de Córdoba (UNC), Argentina. Dirección: Rondeau $4671^{\circ}$ piso. Córdoba, Argentina. CP: 5000. Teléfono: +54-351-4341124. Correo electrónico: ldacosta@ conicet.gov.ar; laudeac@hotmail.com 


\section{Resumen}

Objetivo: analizar las características de la dieta en niños argentinos de 2 a 5 años que reciben intervenciones alimentarias en el hogar, en el año 2005. Métodos: se analizó la Encuesta Nacional de Nutrición y Salud (ENNys). El muestreo de la EnNys fue probabilístico, estratificado por conglomerados, $\mathrm{n}=7046$. Se utilizó la metodología Propensity Score Matching (PSM) para la constitución de grupos comparables de niños que reciben y no reciben intervenciones alimentarias en el hogar. Se analizó el consumo de macro y micronutrientes, energía, proporción de energía destinada a cada tiempo de comida y grupos de alimentos. Resultados y conclusiones: se observó un menor consumo de energía y calcio, y una mayor proporción de energía proveniente de cereales, en los niños que reciben intervención alimentaria en el hogar; lo que indicaría que los niños que reciben programas alimentarios no presentan una mejor calidad de la dieta.

Palabras clave: programas y políticas de nutrición y alimentación; niño; puntaje de propensión; dieta; evaluación de programas y proyectos de salud; encuestas nutricionales

\section{Abstract}

Objective: Analyzing the characteristics of the diet in Argentinian children aged 2 to 5, who received alimentary interventions at home during 2005. Methods: We analyzed the National Survey of Nutrition and Health (ENNys). The ENNys had a probabilistic sampling, stratified by conglomerates. $\mathrm{n}=7046$. The Propensity Score Matching (PMS) methodology was used to constitute comparable groups of children who received and did not receive alimentary interventions at home. We analyzed the consumption of macro and micronutrients, energy, energy ratio assigned to each meal time and food groups. Results and Conclusions: We observed a lower consumption of energy and calcium and a higher proportion of energy from cereals in children that received alimentary intervention at home. This would show that children that receive alimentary programs do not present a better quality of their diets.

Keywords: nutrition and diet programs and policies; child; proneness score; diet; health programs and projects assessment; nutritional surveys

\section{Resumo}

Objetivo: analisar as características da dieta de crianças argentinos de 2 até 5 anos que recebem intervenções alimentares em casa, no ano 2005. Métodos: analisou-se o Inquérito Nacional de Nutrição e Saúde (ENNys). A amostragem da ENNys foi probabilístico, estratificado por conglomerados, $n=7046$. Utilizou-se a metodologia Propensity Score Matching (PSM) para a constituição de grupos comparáveis de crianças que recebem e não recebem intervenções alimentares em casa. Analisou-se o consumo de macro e micronutrientes, energia, proporção de energia destinada para cada tempo de refeição e grupos de alimentos. Resultados $e$ conclusões: observou-se menor consumo de energia e cálcio, e maior proporção de energia proveniente de cereais, nas crianças que recebem intervenção alimentar em casa, o que estaria indicando que as crianças que recebem programas alimentares não apresentam melhor qualidade da dieta.

Palavras-chave: programas e políticas de nutrição e alimentares; criança; escore de propensão; dieta; avaliação de programas e projetos de saúde; inquéritos nutricionais 


\section{Introducción}

El derecho a la alimentación ha sido recogido en numerosos tratados y declaraciones internacionales. En 1996 la Cumbre Mundial sobre la Alimentación (1) estableció la meta de reducir a la mitad el número de personas que padecen subnutrición antes de 2015. Asimismo, en la Declaración del Milenio (2), el primer Objetivo de Desarrollo del Milenio consiste en reducir a la mitad tanto la proporción de personas que viven con menos de un dólar al día como la de las personas que padecen hambre, antes del año 2015.

Con la reforma constitucional de 1994, el Estado argentino incorporó a la Constitución Nacional diferentes tratados e instrumentos internacionales de derechos humanos. Por ello, existe la obligación por parte del Estado argentino de respetar, proteger y realizar, es decir, hacer efectivo y facilitar el derecho a la alimentación, en aquellas personas que presentan vulnerabilidad de padecer inseguridad alimentaria, a través de la política alimentaria.

En Argentina, históricamente, las formas de intervención alimentaria en la población infantil se realizan a través de asistencia directa mediante la entrega de leche o por medio de comedores comunitarios, o indirectamente a través de la entrega de módulos alimentarios, y más recientemente, la entrega de tickets o vales para la compra de alimentos a las familias (3). Diversas investigaciones han criticado la política alimentaria implementada en la Argentina en las décadas de los ochenta y noventa, por diversas causas. Entre ellas se encuentra la falta de regularidad en la entrega de las prestaciones alimentarias (4), la superposición de programas nacionales, provinciales, municipales y organizaciones de la sociedad civil (5), la afectación de la comensalidad familiar y la baja calidad nutricional de los programas (6).
A partir del año 2003 se implementa en Argentina el Plan Nacional de Seguridad Alimentaria (PNSA), por parte el Ministerio de Desarrollo Social del Gobierno de la Nación Argentina. Desde el discurso que plantea la normativa del Plan, se observa que este reconoce a la alimentación como un derecho, y establece además cambios positivos en la implementación, como la elaboración de un padrón único que impida la superposición de los programas en los distintos niveles de gobierno. Diversas investigaciones en nuestro país en la última década se han enfocado en el análisis y la evaluación de los programas alimentarios desde la implementación del PNSA (6-9); sin embargo, son escasas aquellas que se han enfocado en evaluar la calidad nutricional de las prestaciones (10-12). Por otra parte, en estos estudios se valoró la dieta de la población infantil que recibía programas alimentarios, sin compararla con la dieta de niños con las mismas condiciones que no recibían intervenciones.

Teniendo en cuenta estos antecedentes, esta investigación pretende responder el siguiente interrogante: ¿La recepción de programas alimentarios por parte de la población infantil vulnerable de Argentina produce efectos positivos en la dieta en relación con la población vulnerable que no recibe programas alimentarios, en el año 2005, luego de la implementación del PNSA?

La hipótesis que guía el trabajo es que la dieta de los niños en situación de vulnerabilidad que reciben programas alimentarios en Argentina, es mejor que si no recibieran intervenciones. Esta investigación se enfoca en la población infantil, ya que la mayor parte de las intervenciones alimentarias se direccionan hacia los menores de seis años, debido a que estos constituyen uno de los grupos más vulnerables desde el punto de vista biológico y social (3). 
La evaluación de la política alimentaria, como toda política social y de salud, constituye un elemento esencial para analizar sus resultados en relación con sus objetivos. Desde el enfoque de investigación cuantitativo, el "estándar de oro" para evaluar el impacto de programas de intervención lo constituyen los diseños experimentales y cuasiexperimentales (13).

Los diseños experimentales consisten, básicamente, en seleccionar al azar de una muestra de individuos, aquellos que constituirían el grupo de intervención del programa y el grupo control. Este tipo de diseño es el más robusto desde el punto de vista metodológico; sin embargo, no es ético llevarlo a cabo, debido a que todos los individuos tendrían las mismas necesidades de participar (13).

Los diseños cuasiexperimentales son aquellos en los cuales no se selecciona a la población de cada grupo al azar, es decir, existe un criterio intencional en la selección de los beneficiarios. Las técnicas metodológicas que se utilizan para evaluar el impacto en esta clase de diseños de evaluación son las técnicas de comparación pareada, las cuales se consideran la alternativa subóptima de un diseño experimental. En los últimos años se ha avanzado en la técnica Propensity Score Matching (PSM) (correspondencia de puntuación de la propensión) (13-15).

Este método se puede llevar a cabo con una simple sección transversal de datos (13). Brevemente, el PSM es una probabilidad condicional que expresa la posibilidad de un participante de ser asignado o seleccionado para recibir un tratamiento o intervención, dadas ciertas características observadas. El objetivo es mejorar el balance de las covariables observadas y recrear una situación similar a un experimento aleatorizado (16-18).
Utilizando la metodología PSM, el presente trabajo tiene como objetivo analizar las características de la dieta en niños de 2 a 5 años, según la recepción de intervenciones alimentarias en el hogar, con base en los datos de la Encuesta Nacional de Nutrición y Salud (ENNys) realizada en el año 2005.

\section{Metodología y fuente de datos}

Este es un estudio transversal y correlacional. Se analizan datos secundarios de la ennys realizada en el año 2005. El universo de estudio corresponde a niños de 2 a 5 años. El muestreo de la ennys fue probabilístico, estratificado por conglomerados a tres etapas, siendo el $n=7046$ niños de 2 a 5 años (19). El rechazo a participar del estudio constituyó el único criterio de exclusión.

La ENNys valoró las siguientes áreas temáticas: caracterización sociodemográfica, estado nutricional, salud del niño/a, salud sexual y reproductiva, desarrollo infantil, cobertura y acceso a servicios de salud, cobertura y acceso a programas e intervenciones alimentarias. Los instrumentos utilizados para la obtención de indicadores en cada área temática fueron: cuestionario estructurado para la obtención de información sociodemográfica, el cual fue respondido por una persona de 18 años o más; y para la valoración del estado nutricional se utilizó cuestionario recordatorio de 24 horas (para la valoración de la dieta) y mediciones antropométricas (peso y talla) (19).

Para cumplimentar el objetivo de este estudio se analizaron, en primer lugar, las variables que presentaron asociación con la recepción de intervenciones alimentarias en el hogar (variable dependiente), clasificadas en a) variables sociodemográficas: sexo y edad (en meses) del niño/a, región de residencia (Gran Buenos Aires, Cuyo, Noreste, Noroeste, Pampeana, Patagonia), índice de necesidades 
básicas insatisfechas (NBI, categorizado en: sin NBI, al menos un indicador NBI), línea de pobreza/indigencia del hogar (hogar no pobre, hogar pobre no indigente, hogar indigente), sexo del jefe de hogar, nivel educativo del jefe de hogar (bajo: menos de 7 años de estudios; medio: entre 7 y 11 años; alto: 12 años o más), cobertura médica del jefe de hogar (obra social y/o cobertura médica privada, solo pública); b) programas e intervenciones alimentarias en el niño: recepción de leche, asistencia a comedor comunitario; c) estado nutricional: Se construyeron indicadores con base en el peso y la talla de los niños, comparados con las referencias de la Organización Mundial de la Salud (oms). Los indicadores considerados fueron: peso para la edad (bajo peso: niño/a con peso igual o menor a -2 puntajes $\mathrm{Z}$ para su sexo y edad; normalidad: niño/a con peso mayor a -2 puntajes Z), peso para la talla (emaciación: niño/a con peso igual o menor a -2 puntajes $\mathrm{Z}$ para su talla y sexo; obesidad: niño/a con peso igual o mayor a +2 puntajes $\mathrm{Z}$ para su talla y edad; normalidad: niño/a con peso mayor a -2 y menor a +2 puntajes $\mathrm{Z}$ ), talla para la edad (acortamiento: niño/a con talla igual o menor a -2 puntajes $\mathrm{Z}$ para su sexo $\mathrm{y}$ edad; normalidad: niño/a con talla mayor a -2 puntajes $\mathrm{Z}$ para su sexo y edad).

Se excluyeron todos los casos que presentaran valores perdidos en alguna de las variables analizadas, por lo cual la muestra final fue de 5400 niños de 2 a 5 años.

Para el análisis de la asociación entre la variable dependiente "intervención alimentaria en el hogar" y las variables independientes, se analizó a través de análisis de regresión logística binaria o prueba T de Student, de acuerdo con la naturaleza de la variable dependiente. Se aceptó un nivel de significación menor a 0,05 para rechazo de hipótesis nula.
Luego de determinar las variables que se asociaron con la recepción de intervenciones alimentarias en el hogar, se utilizó la metodología Propensity Score Matching (PSM), a los fines de constituir grupos comparables. El PSM se define como la probabilidad de recibir un tratamiento o intervención, basado en la medición de covariables:

$$
e(x)=P(Z=1 \mid \mathrm{X})
$$

Donde $e(x)$ es la abreviación del PSM, P es una probabilidad, $Z=1$ es un indicador de tratamiento, siendo de 0 para el grupo control y de 1 para tratamiento. El símbolo "|" indica condicional de, mientras que $\mathrm{X}$ es un grupo de covariables observadas. Es decir, que el PSM indica la probabilidad de una persona de ser seleccionada para una intervención o tratamiento dadas determinadas variables observadas. Para ello el procedimiento otorga a cada caso un determinado score (puntaje). Este score permite emparejar participantes del grupo de intervención con participantes de la condición de control que poseen similar score.

Este proceso de emparejamiento crea un balance entre el grupo intervenido y el no intervenido a través del score y, fundamentalmente, también se espera que permita crear un balance entre las covariables que son utilizadas para crear el PSM. Este balance es un elemento clave ya que se neutraliza el efecto de confusión de una covariable (16).

Para el cálculo del PSM se cumplimentaron los siguientes pasos (16):

1. Se seleccionó un conjunto de covariables que se presumen importantes basadas en argumentos teóricos. Para ello, se seleccionaron las variables sociodemográficas, de asistencia alimentaria y estado nutricional, que presentaron 
asociación significativa con la variable intervención alimentaria en el hogar.

2. Para el cálculo del score (puntaje) se utilizó regresión logística, en la cual el tratamiento asignado es utilizado como variable de resultado y el grupo de covariables como predictoras. La regresión logística se especifica como:

$$
\operatorname{Ln}\left[\frac{P\left(Z=1 \mid X_{1}, \ldots, X_{j}\right)}{1-P\left(Z=1 \mid X_{1}, \ldots, X_{j}\right)}\right]=\beta_{0}+\sum_{j=1}^{p} \beta_{j} X_{j}
$$

Donde $\mathrm{Z}$ es la variable binaria que indica la condición de intervención o control, y $\mathrm{X}_{1}, \mathrm{X}_{\mathrm{j}}$ son todas las covariables que están siendo usadas para predecir la pertenencia al grupo de intervención o control. Los valores predichos en esta ecuación son los puntajes estimados.

3. Después de la estimación del PSM se produce el procedimiento de emparejamiento. Se utilizó la técnica de emparejamiento 1:2 vecino más cercano, lo cual significa que cada caso que recibe intervención alimentaria en el hogar, es emparejado con dos controles (casos que no reciben intervención alimentaria en el hogar), respectivamente, los cuales poseen el puntaje más cercano. Para asegurar buenos emparejamientos se definió un calibrador (diferencia máxima permitida entre dos participantes), el cual es definido en unidades de desviaciones estándar del logit del puntaje estimado. Para excluir malos agrupamientos se utilizó un calibrador de 0,15 de la desviación estándar del logit del PSM.

4. Después del emparejamiento se llevaron a cabo una serie de modelos de verificación para chequear que el balance de las covariables haya mejorado verdaderamente a través del proceso de emparejamiento. Para ello se utilizó el indicador general de imbalance multivariado $\left(L_{1}\right)$. El indicador $L_{1}$ es definido como (18):

$$
L_{1}=\frac{1}{2} \sum_{1_{1} \ldots l_{k}}\left|t l_{1} \ldots l_{k}-c l_{1} \ldots l_{k}\right|
$$

Donde l es la frecuencia de una determinada celda indicada como $1 \mathrm{a} \mathrm{k}$ de una tabla de contingencia multivariada, para cada grupo intervención (t) y control (c). El test evalúa simultáneamente la distribución conjunta de todas las covariables, lo cual significa que utiliza polinomios de alto grado e interacciones. Si la medición $\mathrm{L}_{1}$ es cero indica balance perfecto, mientras que si es igual a 1 indica disbalance, es decir, separación completa en la tabla de contingencia. No hay un punto de corte que indique mejor o peor balance, pero la situación deseable es que la medición $L_{1}$ sea menor en la muestra emparejada que en la muestra no agrupada.

Además de este test multivariado, se calcularon para cada una de las covariables diferencias de medias estandarizadas, definidas como las medias de las diferencias entre los grupos, dividas por la desviación estándar del grupo control. La diferencia de medias estandarizadas de las covariables debe ser lo más cercana a 0 después del emparejamiento.

El análisis estadístico se realizó con el programa Psmatching (17), el cual realiza el análisis PSM con el programa estadístico R, utilizando el entorno del programa estadístico SPSS.

Para comparar las características de la dieta en relación con la recepción de intervenciones alimentarias en el hogar, se analizó la nueva submuestra obtenida del procedimiento PSM. Se consideró como variable independiente las intervenciones alimentarias y como variables dependientes los indicadores de la 
dieta: consumo de macro y micronutrientes, tiempo de comida y grupos de alimentos.

Los macro y micronutrientes considerados fueron: proteínas (g), grasas saturadas, poliinsaturadas $(\mathrm{g})$, fibra $(\mathrm{g})$, colesterol $(\mathrm{mg})$, vitamina A (mg RAE), vitamina C (mg), vitamina B1 (mg), vitamina B2 (mg), vitamina B12 (mg), niacina $(\mathrm{mg})$, folatos $(\mathrm{mg})$, calcio (mg), hierro (mg) y zinc (mg). Se consideró además la energía (Calorías).

Los valores de cada nutriente fueron comparados con el Requerimiento Promedio Estimado (RPE), según edad (20). Luego fueron categorizados en: adecuado (igual o mayor al RPE)/inadecuado (menor al RPE).

El tiempo de comida categoriza la proporción de energía destinada al desayuno, almuerzo, merienda, cena y colaciones, mientras que los grupos de alimentos considerados agrupan la proporción de energía consumida en lácteos, carnes y huevos, hortalizas y frutas, cereales y legumbres, azúcares y bebidas, grasas y aceites. Toda la información sobre las características de la dieta fue obtenida de cuestionario recordatorio de 24 horas.

Se realizó análisis bivariado: test de $X^{2}$, Odds Ratio (OR), prueba T de Student, de acuerdo con la naturaleza de la variable. Además, se calcularon intervalos de confianza (IC); aceptándose un $\mathrm{p}<0,05$ para rechazo de hipótesis nula. El cálculo estadístico se realizó con el software estadístico spss versión 19.

\section{Resultados}

\section{Descripción de la variable dependiente}

El 25,5\% de los niños recibieron al menos una intervención alimentaria en el hogar. La modalidad de entrega más utilizada fue el módulo de alimentos $(21,7 \%)$, seguida de la entrega de tickets/vales para la compra de alimentos $(4,2 \%)$, y un bajo porcentaje $(1,0 \%)$ recibió la intervención alimentaria con otro tipo de modalidad.

\section{Constitución de grupos comparables}

En primer lugar, se analizó la relación entre la recepción de intervenciones alimentarias en el hogar y las variables independientes. En la tabla 1 se observa que la mayoría de las variables independientes seleccionadas presentaron asociación significativa con la variable dependiente intervención alimentaria en el hogar: peso para la edad (mayor proporción de recepción en niños/as con bajo peso), peso para la talla (menor proporción en niños/as que presentan obesidad), talla para la edad (mayor proporción en niños/as que presentan acortamiento), región de residencia del niño (menor recepción en la región Cuyo), línea pobreza/indigencia (mayor proporción de recepción de intervención alimentaria en hogares pobres y hogares indigentes), hogar NBI (mayor recepción en hogares con NBI), sexo del jefe (mayor proporción de recepción en hogares con jefatura femenina), nivel educativo del jefe (mayor recepción en hogares cuyos jefes presentan nivel educativo bajo y medio), cobertura médica del jefe (mayor recepción de intervenciones alimentarias en hogares cuyo jefe no posee cobertura médica privada u obra social), mayor proporción de recepción de intervención alimentaria en el hogar en niños que reciben leche y en niños que concurren a comedores comunitarios.

En segundo lugar, se aplicó el PSM en la muestra de niños de 2 a 5 años para la variable intervención alimentaria en el hogar. Se introdujeron las covariables (es decir, todas aquellas que podrían explicar la pertenencia al grupo de intervención y control), excepto las que no presentaron asociación significativa con la variable intervención alimentaria 
TABLA 1. VARIABLES SOCIODEMOGRÁFICAS (SEXO, EDAD, REGIÓN DE RESIDENCIA, LÍNEA DE POBREZA/INDIGENCIA, NECESIDADES BÁSICAS INSATISFECHAS, SEXO, EDAD, NIVEL EDUCATIVO Y COBERTURA MÉdICA DEL JEFE DE HOGAR), ASISTENCIA ALIMENTARIA (RECEPCIÓN DE LECHE, ASISTENCIA A COMEDOR COMUNITARIO) Y ESTADO NUTRICIONAL (PESO/EDAD, TALLA/EDAD Y PESO/TALLA) EN NIÑOS/AS DE 2 A 5 AÑOS, SEGÚN RECEPCIÓN DE INTERVENCIÓN alimentaria en el hogar. Argentina, Encuesta Nacional de Nutrición y Salud (ennys), 2005 ( $=5400)$

\begin{tabular}{|c|c|c|c|c|}
\hline Variable & Categorías & Recibe & No recibe & Sig. \\
\hline \multirow{2}{*}{ Sexo del niño } & Femenino & $646(46,8 \%)$ & $1985(49,4 \%)$ & - \\
\hline & Masculino & $734(53,2 \%)$ & $2035(50,6 \%)$ & 0,080 \\
\hline Edad del niño & (Media y DE) & $47,5( \pm 13,8)$ & $47,6( \pm 13,9)$ & 0,665 \\
\hline \multirow{3}{*}{$\begin{array}{l}\text { Peso para la talla } \\
\text { del niño/a }\end{array}$} & Normalidad* & $1246(90,3 \%)$ & $3561(88,6 \%)$ & - \\
\hline & Emaciación & $27(2,0 \%)$ & $61(1,5 \%)$ & 0,314 \\
\hline & Obesidad & $107(7,8 \%)$ & $398(9,9 \%)$ & 0,020 \\
\hline \multirow{2}{*}{$\begin{array}{l}\text { Talla para la } \\
\text { edad del niño/a }\end{array}$} & Normalidad & $1257(91,1 \%)$ & $3821(95,2 \%)$ & \multirow{2}{*}{$<0,001$} \\
\hline & Acortamiento & $123(8,9 \%)$ & $191(4,8 \%)$ & \\
\hline \multirow{2}{*}{$\begin{array}{l}\text { Peso para la } \\
\text { edad del niño/a }\end{array}$} & Normalidad & $1344(97,1 \%)$ & $3956(98,4 \%)$ & \multirow{2}{*}{0,022} \\
\hline & & $36(2,6 \%)$ & $64(1,6 \%)$ & \\
\hline \multirow{6}{*}{$\begin{array}{l}\text { Región de } \\
\text { residencia del } \\
\text { niño/a }\end{array}$} & Gran Buenos Aires* & $209(15,1 \%)$ & $514(12,8 \%)$ & - \\
\hline & Cuyo & $70(5,1 \%)$ & $842(20,9 \%)$ & $<0,001$ \\
\hline & Noreste & $247(17,9 \%)$ & $691(17,2)$ & 0,205 \\
\hline & Noroeste & $324(23,5 \%)$ & $667(16,6 \%)$ & 0,142 \\
\hline & Pampeana & $235(17,0 \%)$ & $667(16,6 \%)$ & 0,095 \\
\hline & Patagonia & $295(21,4 \%)$ & $639(15,9)$ & 0,323 \\
\hline \multirow{3}{*}{$\begin{array}{l}\text { Línea de pobre- } \\
\text { za/indigencia }\end{array}$} & Hogar indigente & $626(45,4 \%)$ & $787(19,6 \%)$ & $<0,001$ \\
\hline & Hogar pobre, no indigente & $542(39,3 \%)$ & $1126(28,0 \%)$ & $<0,001$ \\
\hline & Hogar no pobre, no indigente* & $212(15,4 \%)$ & $2107(52,4)$ & - \\
\hline \multirow{2}{*}{$\begin{array}{l}\text { Necesidades } \\
\text { básicas insatis- } \\
\text { fechas }\end{array}$} & Con NBI & $741(53,7 \%)$ & $932(23,2 \%)$ & \multirow[b]{2}{*}{$<0,001$} \\
\hline & Sin NBI & $639(46,3 \%)$ & $3088(76,8 \%)$ & \\
\hline \multirow{2}{*}{ Sexo del jefe } & Masculino & $940(68,1 \%)$ & $3201(79,6 \%)$ & \multirow{2}{*}{$<0,001$} \\
\hline & Femenino & $440(31,9 \%)$ & $819(20,4 \%)$ & \\
\hline Edad del jefe & media (DE) & $38,9( \pm 12,6)$ & $38,5( \pm 11,4)$ & 0,165 \\
\hline \multirow{3}{*}{ Nivel educativo } & Alto* & $210(15,2 \%)$ & $1889(47,0 \%)$ & - \\
\hline & Medio & $846(61,3 \%)$ & $1757(43,7 \%)$ & $<0,001$ \\
\hline & Bajo & $324(23,5 \%)$ & $374(9,3 \%)$ & $<0,001$ \\
\hline \multirow{2}{*}{$\begin{array}{l}\text { Cobertura médi- } \\
\text { ca del jefe }\end{array}$} & Obra social y/o cobertura médica privada & $420(30,4 \%)$ & $2521(62,7 \%)$ & \multirow{2}{*}{$<0,001$} \\
\hline & Solo pública & $960(69,6 \%)$ & $1499(37,3 \%)$ & \\
\hline \multirow{2}{*}{$\begin{array}{l}\text { Recepción de } \\
\text { leche }\end{array}$} & Sí & $450(32,6 \%)$ & $293(7,3 \%)$ & \multirow{2}{*}{$<0,001$} \\
\hline & No & $930(67,4 \%)$ & $3727(97,2 \%)$ & \\
\hline \multirow{2}{*}{$\begin{array}{l}\text { Asiste a come- } \\
\text { dor comunitario }\end{array}$} & Sí & $221(16,0 \%)$ & $134(3,3 \%)$ & \multirow{2}{*}{$<0,001$} \\
\hline & No & $1159(84,0 \%)$ & $3886(96,7 \%)$ & \\
\hline \multicolumn{5}{|c|}{ Referencias: Sig. $=$ Nivel de significación. $*=$ categoría de referencia. $(D E)=$ desvío estándar } \\
\hline
\end{tabular}

Fuente: elaboración propia con base en la ENNys 2005 
en el hogar (sexo y edad del niño, edad del jefe de hogar. Ver tabla 1).

De un total de 7046 casos de la muestra, se agruparon 1702 controles y 1190 casos. La medición de imbalance multivariado $\left(L_{1}\right)$ fue mayor en la muestra no agrupada $(0,693)$ que después del agrupamiento $(0,531)$.

La figura 1 muestra el balance de las covariables a través de la diferencia de medias estandarizadas de cada una de estas. Se observa que las diferencias de medias estandarizadas luego del agrupamiento fueron iguales o cercanas a cero, es decir, que ambos grupos son comparables, ya que presentan la misma distribución de las variables independientes.

\section{Comparación de las características de la dieta en niños que reciben y que no reciben programas alimentarios}

En primer lugar, se analiza el consumo de macronutrientes, micronutrientes y energía, según la recepción de programas alimen-

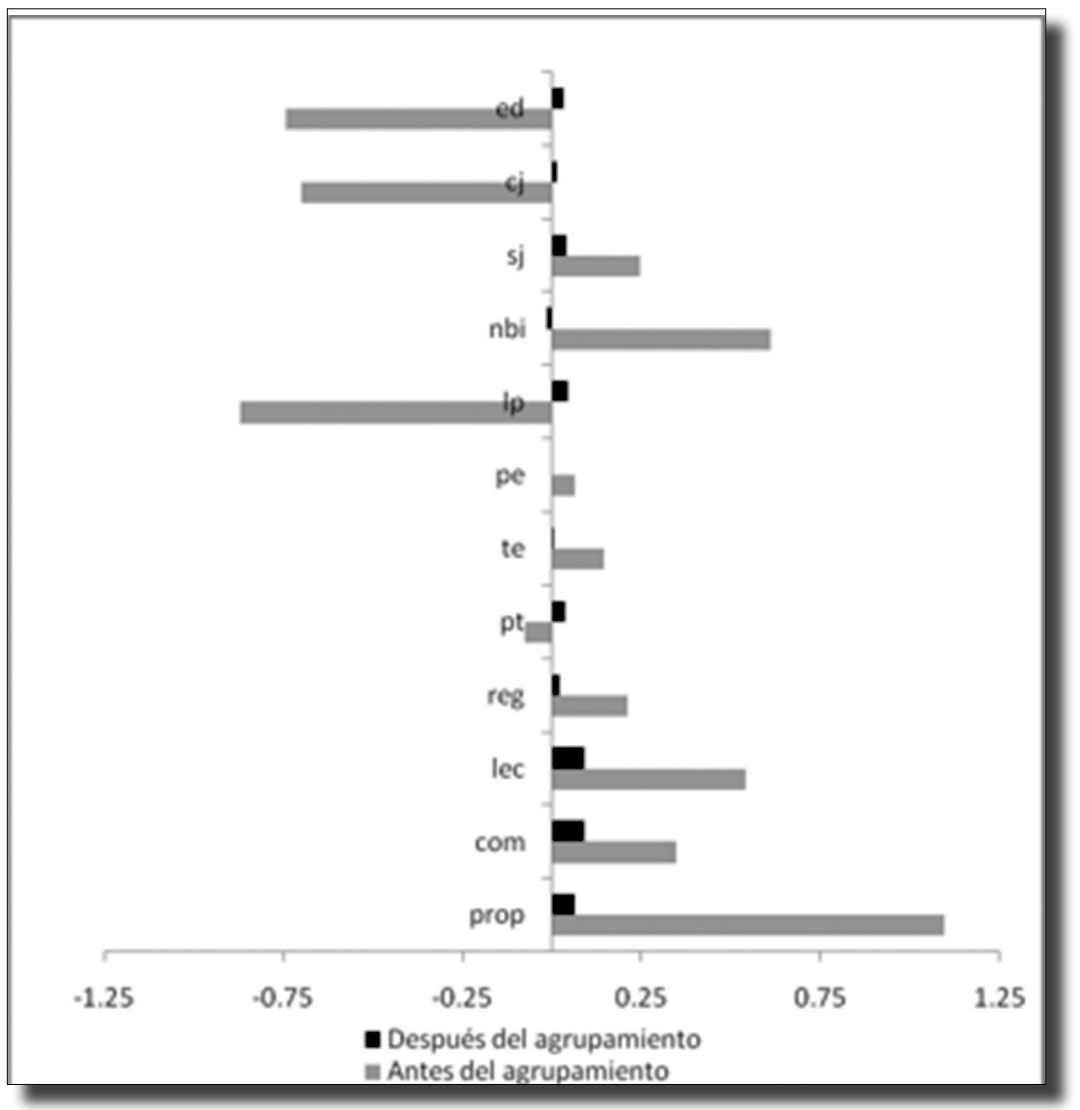

Figura 1. Diferencia de medias estandarizadas (COHEN`s $D$ ) de todas las Covariables Consideradas, antes y después del agrupamiento. Niños de 2 a 5 años; Argentina ennys, 2005

Referencias: prop (propensity), com (niño concurre a comedor comunitario), lec (niño recibe leche),reg (región de residencia), pt (peso para la talla), te (talla para la edad), pe (peso para la edad), lp (línea de pobreza/indigencia), nbi (necesidades básicas insatisfechas), sj (sexo del jefe de hogar), cj (cobertura médica del jefe de hogar), ed (nivel educativo del jefe de hogar).

Fuente: elaboración propia con base en la ENNys 2005 
tarios en el hogar. Se observa, en relación con la ingesta de nutrientes, una menor proporción de ingesta adecuada de calcio en niños que provienen de hogares que reciben al menos una intervención alimentaria en el hogar. El resto de los nutrientes no presentaron diferencias significativas. Por otra parte, se observa una menor proporción de ingesta adecuada de energía (tabla 2 ).
Respecto al tiempo de comida y los grupos de alimentos, en la tabla 3 se observa que no existieron diferencias en la proporción de energía proveniente de cada tiempo de comida. En relación con los grupos de alimentos se observa una mayor proporción de energía proveniente de cereales y una menor proporción de energía proveniente de dulces y bebidas en niños que reciben intervención alimentaria en el hogar, en comparación con niños que no reciben.

TABLA 2. Proporción de ingesta Adecuada/INAdecuada de MACronutrientes, Micronutrientes y ENERGía,

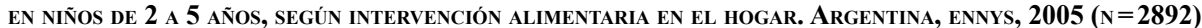

\begin{tabular}{|c|c|c|c|c|c|c|c|}
\hline \multirow{2}{*}{ Nutriente } & \multirow{2}{*}{$\begin{array}{l}\text { Intervención } \\
\text { alimentaria en } \\
\text { el hogar* }\end{array}$} & \multirow{2}{*}{$\begin{array}{l}\text { Ingesta ade- } \\
\text { cuada }\end{array}$} & \multirow{2}{*}{$\begin{array}{l}\text { Ingesta inade- } \\
\text { cuada }\end{array}$} & \multirow{2}{*}{ Sig. } & \multirow{2}{*}{$\mathrm{OR}^{* *}$} & \multicolumn{2}{|c|}{ IC $95 \%$} \\
\hline & & & & & & Inferior & Superior \\
\hline \multirow{2}{*}{ Energía (Cal) } & No recibe & $1271(74,7 \%)$ & $431(25,3 \%)$ & \multirow{2}{*}{0,012} & \multirow{2}{*}{1,239} & \multirow{2}{*}{1,050} & \multirow{2}{*}{1,462} \\
\hline & Recibe & $838(70,4 \%)$ & $352(29,6 \%)$ & & & & \\
\hline \multirow{2}{*}{ Proteínas (g) } & No recibe & $1674(98,4 \%)$ & $28(1,6 \%)$ & \multirow{2}{*}{0,195} & \multirow{2}{*}{0,609} & \multirow{2}{*}{0,308} & \multirow{2}{*}{1,203} \\
\hline & Recibe & $1178(99,0 \%)$ & $12(1,0 \%)$ & & & & \\
\hline \multirow{2}{*}{ Fibra (g) } & No recibe & $63(3,7 \%)$ & $1638(96,3 \%)$ & \multirow{2}{*}{0,438} & \multirow{2}{*}{0,857} & \multirow{2}{*}{0,588} & \multirow{2}{*}{1,249} \\
\hline & Recibe & $51(4,3 \%)$ & $1136(95,7 \%)$ & & & & \\
\hline \multirow{2}{*}{$\begin{array}{l}\text { Ácidos grasos } \\
\text { saturados (g) }\end{array}$} & No recibe & $611(35,9 \%)$ & $1091(64,1 \%)$ & \multirow{2}{*}{0,433} & \multirow{2}{*}{0,938} & \multirow{2}{*}{0,804} & \multirow{2}{*}{1,093} \\
\hline & Recibe & $445(37,4 \%)$ & $745(62,6 \%)$ & & & & \\
\hline \multirow{2}{*}{$\begin{array}{l}\text { Ácidos grasos } \\
\text { poliinsat. (g) }\end{array}$} & No recibe & $508(29,8 \%)$ & $1194(70,2 \%)$ & \multirow{2}{*}{0,590} & \multirow{2}{*}{1,046} & \multirow{2}{*}{0,889} & 1921 \\
\hline & Recibe & $344(28,9 \%)$ & $846(71,1 \%)$ & & & & \\
\hline Colesterol & No recibe & $1445(84,9 \%)$ & $257(15,1 \%)$ & 0750 & 0062 & 0700 & 1106 \\
\hline (mg) & Recibe & $1016(85,4 \%)$ & $174(14,6 \%)$ & 0,130 & 0,905 & $0, / 02$ & 1,100 \\
\hline & No recibe & $1595(93,7 \%)$ & $107(6,3 \%)$ & & & & \\
\hline TOIatos (ing) & Recibe & $1120(94,1 \%)$ & $70(5,9 \%)$ & 0,094 & 0,932 & 0,003 & $1,2 / 1$ \\
\hline Vitamina $\mathrm{C}$ & No recibe & $647(38,0 \%)$ & $1055(62,0 \%)$ & - 707 > & 007 & 0006 & 190 \\
\hline (mg) & Recibe & $460(38,7 \%)$ & $730(61,3 \%)$ & 0,121 & 0, & 0,030 & $1,15 J$ \\
\hline N. & No recibe & $1576(92,6 \%)$ & $126(7,4 \%)$ & 0715 & 0000 & 0700 & 1005 \\
\hline INIacinda (ing) & Recibe & $1107(93,0 \%)$ & $83(7,0 \%)$ & 0,113 & 0,950 & $0, / 03$ & $1,20 \mathrm{~J}$ \\
\hline Rivoflavina & No recibe & $1673(98,3 \%)$ & $29(1,7 \%)$ & & & & \\
\hline$(\mathrm{mg})$ & Recibe & $1164(97,8 \%)$ & $26(2,2 \%)$ & , & & & 2,10 \\
\hline$\pi \cdot$. & No recibe & $1659(97,5 \%)$ & $43(2,5 \%)$ & ה 72 & 100 & 0605 & 174 \\
\hline 1 Hanmina (ing) & Recibe & $1157(97,2 \%)$ & $33(2,8 \%)$ & 0,124 & 1,100 & ט, & $1,74 J$ \\
\hline Vitamina B12 & No recibe & $1615(94,9 \%)$ & $87(5,1 \%)$ & & & & \\
\hline$(\mathrm{mg})$ & Recibe & $1138(95,6 \%)$ & $52(4,4 \%)$ & $0,5 / 0$ & 0,040 & 0,39ו & 1,200 \\
\hline Vitamina A & No recibe & $1162(68,3 \%)$ & $540(31,7 \%)$ & & & & \\
\hline (mg RAE) & Recibe & $793(66,6 \%)$ & $397(33,4 \%)$ & $0,3 / 4$ & $1,0 / 1 /$ & $0,9<0$ & 1,202 \\
\hline
\end{tabular}


CARACTERISTICAS DE LA DIETA EN NIÑOS DE 2 A 5 AÑOS SEGÚN LA RECEPCIÓN DE PROGRAMAS ALIMENTARIOS EN EL hogar en ARgentina

\begin{tabular}{|c|c|c|c|c|c|c|c|}
\hline \multirow{2}{*}{ Nutriente } & \multirow{2}{*}{$\begin{array}{l}\text { Intervención } \\
\text { alimentaria en } \\
\text { el hogar* }\end{array}$} & \multirow{2}{*}{$\begin{array}{l}\text { Ingesta ade- } \\
\text { cuada }\end{array}$} & \multirow{2}{*}{$\begin{array}{l}\text { Ingesta inade- } \\
\text { cuada }\end{array}$} & \multirow[b]{2}{*}{ Sig. } & \multirow[b]{2}{*}{$\mathrm{OR}^{* *}$} & \multicolumn{2}{|c|}{ IC $95 \%$} \\
\hline & & & & & & Inferior & Superior \\
\hline \multirow{2}{*}{ Zinc (mg) } & No recibe & $1614(94,8 \%)$ & $88(5,2 \%)$ & \multirow{2}{*}{0,129} & \multirow{2}{*}{0,754} & \multirow{2}{*}{0,525} & \multirow{2}{*}{1,083} \\
\hline & Recibe & $1143(96,1 \%)$ & $47(3,9 \%)$ & & & & \\
\hline \multirow{2}{*}{ Calcio (mg) } & No recibe & $947(55,6 \%)$ & $755(44,4 \%)$ & \multirow{2}{*}{0,012} & \multirow{2}{*}{1,209} & \multirow{2}{*}{1,042} & \multirow{2}{*}{1,402} \\
\hline & Recibe & $606(50,9 \%)$ & $584(49,1 \%)$ & & & & \\
\hline \multirow{2}{*}{ Hierro (mg) } & No recibe & $1628(95,7 \%)$ & $74(4,3 \%)$ & \multirow{2}{*}{0,926} & \multirow{2}{*}{0,965} & \multirow{2}{*}{0,669} & \multirow{2}{*}{1,392} \\
\hline & Recibe & $1140(95,8 \%)$ & $50(4,2 \%)$ & & & & \\
\hline \multicolumn{8}{|c|}{ No recibe $n=1702$ Recibe $n=1190$} \\
\hline
\end{tabular}

Fuente: elaboración propia con base en la EnNys 2005

TABLA 3. Diferencia de Medias de La PROPORCIÓN DE ENERgía de CADA TIEMPO dE COMIDA Y PROPORCIÓN DE ENERGÍA DE CADA GRUPO DE ALIMENTOS CONSUMIDOS, EN NIÑOS DE 2 A 5 AÑOS, SEGÚN INTERVENCIÓN ALIMENTARIA En El hogar. Argentina ennys, 2005 ( $\mathrm{N}=\mathbf{2 8 9 2})$

\begin{tabular}{|c|c|c|c|c|c|c|c|}
\hline \multirow{2}{*}{ Variable } & \multirow{2}{*}{$\begin{array}{l}\text { Intervención alimen- } \\
\text { taria en el hogar* }\end{array}$} & \multirow{2}{*}{ Media } & \multirow{2}{*}{$\mathrm{DE}$} & \multirow{2}{*}{ Sig. } & \multirow{2}{*}{$\begin{array}{l}\text { Dif. de } \\
\text { medias }\end{array}$} & \multicolumn{2}{|l|}{ IC $95 \%$} \\
\hline & & & & & & Inferior & Superior \\
\hline \multirow{2}{*}{$\begin{array}{l}\text { Energía desayuno (pro- } \\
\text { porción) }\end{array}$} & No recibe & 0,192 & 0,099 & \multirow{2}{*}{0,112} & \multirow{2}{*}{$-0,006$} & \multirow{2}{*}{$-0,013$} & \multirow{2}{*}{0,001} \\
\hline & Recibe & 0,198 & 0,101 & & & & \\
\hline \multirow{2}{*}{$\begin{array}{l}\text { Energía almuerzo (pro- } \\
\text { porción) }\end{array}$} & No recibe & 0,288 & 0,130 & \multirow{2}{*}{0,221} & \multirow{2}{*}{0,006} & \multirow{2}{*}{$-0,004$} & \multirow{2}{*}{0,016} \\
\hline & Recibe & 0,282 & 0,132 & & & & \\
\hline \multirow{2}{*}{$\begin{array}{l}\text { Energía merienda (pro- } \\
\text { porción) }\end{array}$} & No recibe & 0,202 & 0,115 & \multirow{2}{*}{0,542} & \multirow{2}{*}{$-0,003$} & \multirow{2}{*}{$-0,011$} & \multirow{2}{*}{0,006} \\
\hline & Recibe & 0,205 & 0,112 & & & & \\
\hline \multirow{2}{*}{$\begin{array}{l}\text { Energía cena (propor- } \\
\text { ción) }\end{array}$} & No recibe & 0,199 & 0,126 & \multirow{2}{*}{0,750} & \multirow{2}{*}{0,002} & \multirow{2}{*}{$-0,008$} & \multirow{2}{*}{0,011} \\
\hline & Recibe & 0,197 & 0,128 & & & & \\
\hline \multirow{2}{*}{$\begin{array}{l}\text { Energía colaciones } \\
\text { (proporción) }\end{array}$} & No recibe & 0,120 & 0,137 & \multirow{2}{*}{0,842} & \multirow{2}{*}{0,001} & \multirow{2}{*}{$-0,009$} & \multirow{2}{*}{0,011} \\
\hline & Recibe & 0,119 & 0,142 & & & & \\
\hline \multirow{2}{*}{$\begin{array}{l}\text { Energía (leche, queso, } \\
\text { yogur) (proporción) }\end{array}$} & No recibe & 0,210 & 0,146 & 0161 & 0008 & 0002 & 0018 \\
\hline & Recibe & 0,203 & 0,143 & 0,101 & 0,008 & $-0,003$ & 0,018 \\
\hline Energía (carnes, pesca- & No recibe & 0,116 & 0,091 & 0 & 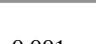 & - & م \\
\hline do, huevo) (proporción) & Recibe & 0,115 & 0,092 & 0,698 & 0,001 & $-0,005$ & 0,008 \\
\hline Energía (hortalizas, & No recibe & 0,069 & 0,067 & 0757 & 0001 & 0004 & 0006 \\
\hline frutas) (proporción) & Recibe & 0,069 & 0,067 & 0,757 & 0,001 & $-0,004$ & 0,006 \\
\hline Energía (cereales, legu- & No recibe & 0,340 & 0,157 & 0010 & 0014 & 0026 & 0002 \\
\hline minosas) (proporción) & Recibe & 0,354 & 0,157 & 0,019 & $-0,014$ & $-0,0 \angle 0$ & $-0,002$ \\
\hline Energía (aceites, grasas) & No recibe & 0,085 & 0,070 & 0432 & 0002 & -0007 & 0003 \\
\hline (proporción) & Recibe & 0,087 & 0,073 & 0,432 & $-0,002$ & $-0,007$ & 0,003 \\
\hline Energía (dulces, bebi- & No recibe & 0,179 & 0,102 & 0030 & 0008 & 0000 & 0015 \\
\hline das) (proporción) & Recibe & 0,171 & 0,100 & 0,039 & 0,008 & 0,000 & 0,015 \\
\hline
\end{tabular}

*No recibe $\mathrm{n}=1702$ Recibe $\mathrm{n}=1190$.

Referencias = Sig: nivel de significación; ic: intervalo de confianza; de: desvío estándar

Fuente: elaboración propia con base en la ENNys 2005 
Por lo tanto, en líneas generales, no existieron grandes diferencias en la calidad de la dieta en niños que reciben y que no reciben programas alimentarios, e incluso se observan aspectos negativos en los primeros, como un menor consumo de calcio y energía.

\section{Discusión}

El presente trabajo es el primer estudio realizado en Argentina que evalúa globalmente la calidad nutricional de los programas alimentarios en la población de 2 a 5 años y además es el segundo trabajo que utiliza la metodología PSM para el análisis de programas alimentarios.

En primer lugar, se observó que la mayor proporción de niños que recibían intervenciones alimentarias fue a través de caja/ bolsa de alimentos. Esta modalidad es la más utilizada históricamente en Argentina (3), y solo en los últimos años se ha difundido más ampliamente el uso de tarjetas magnéticas o vales para la compra de alimentos; propuesta que ha presentado experiencias exitosas en la región de América Latina, como los programas "Bolsa Escola" y "Hambre Cero" en Brasil, "Asignaciones Familiares" en Honduras, o "Progresa" en México (21).

Se observó que existieron diferencias significativas en la población de niños de 2 a 5 años que reciben programas alimentarios, en relación con la mayoría de las variables independientes analizadas (variables sociodemográficas, estado nutricional y recepción de otras intervenciones alimentarias). Estas asociaciones eran esperables, ya que los programas alimentarios están enfocados a la población vulnerable, desde el punto de vista biológico y social: niños, embarazadas y ancianos de bajo nivel socioeconómico. Sin embargo, cabe destacar que si bien un alto porcentaje de niños vivían en hogares en condiciones de vulnerabilidad (hogares pobres, indigentes y/o con NBI), una elevada proporción no recibieron intervenciones alimentarias, siendo la cobertura de programas alimentarios en hogares vulnerables inferior al 50\%. A este respecto Díaz (22) sostiene que existe un "desencuentro" entre la población vulnerable y la cobertura de la política alimentaria. De acuerdo con este autor, teniendo en cuenta la magnitud del problema alimentario en Argentina, resulta casi imposible responder de manera adecuada a través de programas nutricionales focalizados, por lo que estas acciones asistenciales deben ir acompañadas de la complementación de intervenciones que promuevan de manera universal, continua y permanente el acceso a una alimentación adecuada.

Por otra parte, existen diferentes criterios de focalización en relación con los términos de "vulnerabilidad" o "riesgo nutricional" establecidos en los requisititos para acceder a un programa alimentario, los cuales varían de un programa al otro (23). En este sentido, se destaca que el PNSA indique claramente que se establece como vulnerable el criterio de pobreza establecido por el Instituto Nacional de Estadísticas y Censos (9).

Respecto a los resultados observados acerca de la calidad de la dieta en niños destinatarios y no destinatarios de programas alimentarios, contrariamente a la hipótesis planteada, los niños provenientes de hogares vulnerables no presentaron una mejor calidad de la dieta, e incluso se observaron algunos aspectos negativos entre los niños destinatarios, como una menor proporción de consumo adecuado de calcio y de energía.

En estudios precedentes se había detectado que la dieta de los niños que reciben programas alimentarios no cubren las recomendaciones de nutrientes críticos en Argentina, como el calcio (10-12). En este 
trabajo se observó, además, que el consumo de calcio fue menor en niños que reciben programas alimentarios que en niños con las mismas condiciones de vulnerabilidad que no reciben intervenciones alimentarias en el hogar. Este hecho se observó también en la ingesta de energía, lo cual requeriría nuevos estudios para analizar el porqué de una peor manifestación de la adecuación de estos indicadores en los niños que reciben intervenciones alimentarias en el hogar.

Otra característica de la dieta en la cual se registraron diferencias fue en una mayor proporción de energía en la dieta proveniente de cereales y leguminosas en niños que reciben intervenciones alimentarias. Como se indicara anteriormente, la intervención alimentaria más frecuente ha sido la entrega de módulos y cajas de alimentos, en los cuales el principal grupo de alimentos son los cereales y derivados (3), y de allí se explica la mayor proporción de consumo de este grupo de alimentos. El aporte de nutrientes de los programas alimentarios, en especial a través de módulos o cajas de alimentos, ha sido criticado por numerosos estudios (6-8) debido a su baja calidad nutricional, teniendo en cuenta sobre todo el aporte de hidratos de carbono refinados.

Como aspecto positivo, en los niños que reciben programas alimentarios se observó una menor proporción de energía proveniente de azúcares y bebidas. Es posible que esta característica positiva se encuentre relacionada con la educación alimentaria nutricional que aportan algunos programas alimentarios en el marco del PNSA (21).

Otros estudios han evaluado el impacto de programas alimentarios en la calidad de la dieta a través de la metodología PSM. Rawat et ál. (24) evaluaron el impacto de un programa alimentario destinado a población con VIH y destacaron que — al igual que en el pre- sente trabajo- no se observaron diferencias en la calidad de la dieta, aunque se lograron cambios en otros indicadores de impacto como el estado nutricional antropométrico o la concentración de hemoglobina.

En otro estudio realizado en Estados Unidos se evalúo la calidad de la dieta del programa de alimentación escolar. Los autores destacan que los niños que participaron del programa - contrariamente a lo observado en esta investigación- presentaron menor prevalencia de ingesta inadecuada de nutrientes, aunque se registró una mayor proporción de ingesta excesiva de sodio (25).

Otros estudios han evaluado programas alimentarios utilizando la metodología PSM, aunque han utilizado otros indicadores de impacto, como el estado nutricional antropométrico. Así, Leroy et ál. (26) evaluaron el programa de transferencia condicionada de dinero "Oportunidades", desarrollado en México, el cual tiene un fuerte componente alimentario. Utilizando la metodología PSM, los autores observaron que se relacionó positivamente con el crecimiento infantil.

Foster et ál. (27) evaluaron el "Programa de suplementación nutricional especial para embarazadas y niños" de los Estados Unidos, a través de la metodología PSM, y hallaron efectos moderados en algunos de los indicadores de salud de madres y niños.

En Perú se llevaron a cabo dos estudios para evaluar el impacto de programas alimentarios en la población infantil $(15,28)$. El "Programa del vaso de leche" demostró no producir ningún impacto en los niños (15), mientras que el programa "Juntos" evidenció un impacto positivo en la disminución de la desnutrición crónica (28).

En cuanto a las limitaciones del presente estudio, en primer lugar, para la evaluación 
de la dieta la metodología recordatorio de 24 horas presenta ciertas ventajas y desventajas. Entre las desventajas se encuentra que muchas veces no representa el consumo habitual de alimentos y nutrientes de los individuos, en cambio, constituye una buena alternativa para obtener información sobre poblaciones (29).

En relación con la metodología PSM, una limitación es la utilización de un software desarrollado por Thoemmes(17), que utiliza una ventana de dialogo en el entorno spss. En este programa no se utilizan algunos algoritmos de agrupamiento, ni se implementan otras técnicas de evaluación de balance estadístico, que sí se pueden realizar con otros programas como Stata (17).

Por otra parte, la principal limitación se encuentra en la naturaleza transversal de la encuesta, la cual no ha permitido incorporar otras variables de resultado, como el estado nutricional antropométrico y bioquímico en la evaluación de los resultados de los programas. La consideración de estas variables de resultado hubiera permitido un análisis más completo de los efectos de los programas alimentarios. Estas variables han sido observadas en otros estudios de evaluación de programas alimentarios a través de la metodología PSM en Perú $(15,28)$, Estados Unidos (27) y México (26).

Las covariables seleccionadas estuvieron supeditadas a las relevadas por la ENNys. Una variable de interés hubiera sido el tiempo de exposición al programa (15), lo cual hubiese permitido utilizar además otras variables de resultado presentes en la encuesta.

\section{Conclusiones}

En primer lugar, aunque no fue el objetivo de esta investigación, una conclusión de esta es que la cobertura de programas alimentarios en la población infantil vulnerable es baja, por lo que se debería mejorar el acceso a estos, en cumplimiento del derecho a la alimentación.

Con respecto al objetivo planteado en el presente trabajo, como conclusión se destaca que en líneas generales, a pesar de que los programas alimentarios deberían mejorar la alimentación infantil, no se observaron grandes diferencias en la calidad de la dieta entre los niños que reciben y los que no reciben programas alimentarios. Incluso se presentan algunos aspectos negativos en relación con los nutrientes críticos como el consumo de calcio y el consumo de energía entre los destinatarios de estos programas.

Se necesitan nuevas investigaciones para profundizar en las causas de estos hallazgos, para lo cual es necesario contar con información actualizada sobre la situación alimentaria-nutricional de los niños argentinos en situación de vulnerabilidad. A través de la metodología PSM esta evaluación podría realizarse con una encuesta transversal, si se tiene en cuenta en su elaboración la secuencia temporal de las variables analizadas, así como la incorporación de otras variables de interés, como por ejemplo el tiempo de exposición a los programas alimentarios. Asimismo, se podrían incorporar otras técnicas de balance estadístico a la metodología PSM.

A pesar de las limitaciones con respecto a la naturaleza transversal de la encuesta y las variables disponibles, la metodología PSM ha permitido realizar una aproximación a través de la información disponible en Argentina.

\section{Referencias bibliográficas}

1. Food and Agriculture Organization (FAO). Cumbre Mundial de la Alimentación. Roma: Organización de las Naciones Unidas para la Alimentación y la Agricultura; 1996 [Internet] [acceso: 12 de octubre del 2013] Disponible en: http://www.fao.org/wfs/index es.htm

2. Organización de las Naciones Unidas. Öbjetivos de Desarrollo del Milenio. Nueva York: Naciones 
Unidas; 2000 [Internet] [acceso: 12 de octubre del 2013]. Disponible en: http://www.un.org/es/millenniumgoals/bkgd.shtml

3. Britos S, O`Donnell A, Ugalde V, Clacheo R. Programas alimentarios en Argentina. Buenos Aires: Centro de Estudios Sobre Nutrición Infantil (CESNI); 2003.

4. Maceira D, Stechina M. Salud y nutrición. Intervenciones de la política nutricional en 25 años de democracia. Buenos Aires: CIPPEC; 2008.

5. Aguirre P. Seguridad alimentaria: una visión desde la antropología alimentaria. En: Sabulsky J, Ezpeleta ML, Chesta M, Fundación CLACYD, editores. Desarrollo integral de la infancia: El futuro comprometido. Córdoba: CLACYD; 2004. pp. 95-103.

6. O’Donnell A. Iniciativa cuchara, cuchillo y tenedor en casa. Buenos Aires: Centro de Estudios Sobre Nutrición Infantil (CESNI); 2003.

7. Aguirre P. La asistencia social vista por los pobres y nuevos pobres en Buenos Aires durante la convertibilidad. Anthropology of Food; 2009 [Internet] [acceso: 19 de octubre de 2013]. Disponible en: http://aof.revues.org/6439

8. Britos S, Costa R. Seguridad alimentaria nutricional y políticas públicas: el caso argentino 2001-2007. En: FAO. Memoria Concurso red SAN 2007: artículos ganadores. Santiago de Chile: FAO; 2008. pp. 298-325.

9. Garibotti F. Actores, intereses, estrategias y escenarios en el proceso de configuración del programa "Vale lo Nuestro" de la ciudad de Córdoba 2004-2005 [tesis de maestría] Córdoba: Instituto de Investigación y Formación en Administración Pública, Universidad Nacional de Córdoba; 2007.

10. Buamden S, Graciano A, Manzano G, Zummer E. Proyecto "Encuesta a los servicios alimentarios de comedores escolares estatales" (PESCE): alcance de las metas nutricionales de las prestaciones alimentarias de los comedores escolares de Gran Buenos Aires, Argentina. Diaeta. 2010; 28 (130): 21-30.

11. Rovirosa A, Britos S. Estudio sobre la alimentación en niños en edad escolar que concurren a comedores escolares de la provincia de Río Negro-2003[monografía en Internet]. Buenos Aires: Centro de Estudios Sobre Nutrición Infantil (CESNI); 2003 [acceso: 15 de diciembre del 2012]. Disponible en: http://files.cloudpier.net/cesni/biblioteca/a77cda04a73d499417130000.pdf

12. Britos S. Programas alimentarios innovadores: Evaluación de una experiencia en el uso de tarjetas alimentarias [monografía en Internet] Buenos Aires: Centro de Estudios Sobre Nutrición Infantil (CESNI); 2005 [Internet] [acceso: 15 de diciembre de 2012]. Disponible en: http://files.cloudpier.net/ cesni/biblioteca/1adbd703a73d491013070000.pdf

13. Baker JL. Evaluación del impacto de los proyectos de desarrollo en la pobreza. Manual para profesionales. Washington: Banco Mundial; 2005.

14. Jalan J, Ravallion M. Income Gains from Workfare: Estimates for Argentina's Trabajar Program Using Matching Methods. Washington: World Bank; 1998.

15. Gajate Garrido G, Inurritegui Maúrtua M. El impacto de los programas alimentarios sobre el nivel de nutrición infantil: Una aproximación a partir de la metodología del Propensity Score Matching. Lima: CIES/Grade; 2003.

16. Thoemmes FJ, Kim ES. A Systematic Review of Propensity Score Methods in the Social Sciences. Multivariate Behavioral Research. 2008; 46 (1): 90-118.

17. Thoemmes, F. Propensity Score Matching in SPSS [monografía en Internet]. Germany: University of Tübingen; 2012 [acceso: 15 de enero de 2013]. Disponible en: http://arxiv.org/ftp/arxiv/ papers/1201/1201.6385.pdf.

18. Iacus SM, King G, Porro G. CEM: Software for Coarsened Exact Matching. J Stat Soft [serie en Internet] 2009 [acceso: 15 de mayo de 2013] 30 (9) [27 páginas]. Disponible en: http://www.jstatsoft. org/v30/i09/paper

19. Ministerio de Salud de la Nación Argentina. Encuesta Nacional de Nutrición y Salud (ENNys). Documento de resultados. Buenos Aires: Ministerio de Salud de la Nación Argentina; 2007 [Internet] [acceso: 18 de febrero del 2013]. Disponible en: http://www.msal.gov.ar/htm/site/ennys/pdf/ENNys_Documento-de-resultados-2007-II.pdf

20. FAO/WHO. Report of a joint FAO/WHO expert consultation. Human, Vitamin and Mineral Requirements. Bangkok: FAO/WHO; 2001.

21. Aulicino C. Una primera aproximación a las políticas de educación nutricional en las provincias argentinas. Documento de Trabajo no 90 . Buenos Aires: CIPPEC; 2012.

22. Díaz A. Políticas alimentarias: Algo más que programas compensatorios. Ponencia del Congreso: De la Universidad Pública a la Sociedad Argentina. El Plan Fénix en Vísperas del Segundo Centenario; 2005, agosto 2-5. Buenos Aires: FCE/UBA; 2005.

23. Vinocur P, Halperin L. Pobreza y políticas sociales en Argentina de los años noventa. Santiago de Chile: Cepal; 2004.

24. Rawat R, Faust E, Maluccio JA, Kadiyala S. The Impact of a Food Assistance Program on Nutritional Status, Disease Progression, and Food Security among People Living with HIV in Uganda. J Acquir Inmune Defic Syndr 2014; 66 (1): 15-22.

25. Clark MA, Fox MK. Nutritional Quality of the Diets of US Public School Children and the Role of the School Meals Programs. J Am Diet Assoc 2009; 109 (Supp 12): S44-S56.

26. Leroy JL, García-Guerra A, García R, Domínguez C, Rivera J, Neufeld L. The Oportunidades Program Increases the Linear Growth of Children Enrolled at Young Ages in Urban Mexico. J Nutr. 2010; 138 (4): 793-8.

27. Foster EM, Jiang M, Gibson-Davis MC. The Effect of the wIC Program on the Health of Newborns. Health Services Research. 2010; 45 (4): 1083-1104.

28. Sánchez A, Jaramillo Baanante M. Impacto del programa Juntos sobre la nutrición temprana. Revista de Estudios Económicos. 2012 Jun 23; 56-66.

29. Zacarías I. Métodos de evaluación dietética. En: Morón C, Zacarías I, de Pablo S, editores. Producción y manejo de datos de composición química de alimentos en nutrición. Santiago de Chile: FAO; 1997. pp. 91-105. 